\title{
Elaboración de un queso procesado tipo untable obtenido a partir de queso costeño
}

\author{
Margarita R. Arteaga-Márquez, Héctor L. Hernández-Hernández y César D. Peñate-Quiroz \\ Facultad de Ingeniería, Dpto. de Ingeniería de Alimentos, Grupo de Investigación en Procesos Agroindustriales, \\ Universidad de Córdoba, Carrera 6 No. 76-103, Montería, Córdoba, Colombia. \\ (correo-e: mrarteaga@correo.unicordoba.edu.co; hectorhh16@hotmail.com; cespeq@hotmail.com)
}

Recibido Ago. 16, 2019; Aceptado Oct. 11, 2019; Versión final Dic. 11, 2019, Publicado Abr. 2020

\begin{abstract}
Resumen
Esta investigación tuvo como objetivo evaluar un queso procesado untable obtenido de queso costeño (queso fresco), a través de sus características fisicoquímicas, microbiológicas y sensoriales y vida útil. Se usó un diseño experimental al azar, y los datos se procesaron mediante Análisis de varianza y Test de Tukey. Los experimentos fueron por triplicado utilizando mezclas de queso costeño (fresco y envejecido) con diferentes porcentajes $(40,50$ y $60 \%$ ) y distintos tiempos de envejecimiento del queso costeño (15 y 30 días). El contenido de Humedad, Proteína, Acidez y pH obtenido en los quesos fue semejante a otros quesos fundidos, siendo el contenido de grasa y sal superior e inferior, respectivamente. Las características microbiológicas cumplen con lo establecido para este queso. El tratamiento con mayor aceptación sensorial fue el compuesto por $40 \%$ de queso costeño envejecido por 15 días y $60 \%$ de queso costeño fresco, mientras que la vida útil fue de aproximadamente 23 días.
\end{abstract}

Palabras claves: aprovechamiento; vida útil; queso fundido; queso costeño; envejecimiento

\section{Elaboration of a processed cheese spread obtained from costeño cheese}

\begin{abstract}
This research aimed to evaluate a spreadable processed cheese obtained from costeño cheese (fresh cheese), through its physicochemical, microbiological and sensory characteristics and shelf life. A completely randomized experimental design was used, the data were processed by Analysis of variance and Tukey test. The experiments were in triplicate using mixtures of costeño cheese (fresh and aged) with different percentages (40,50 and $60 \%$ ) and different aging times of the costeño cheese (15 and 30 days). The moisture, protein, and acidity content and $\mathrm{pH}$ obtained in the cheeses were similar to other melted cheeses, the fat content and the salt being higher and lower, respectively. The microbiological characteristics are within thiose established for this cheese. The processed cheese with greater sensory acceptance was composed of $40 \%$ of costeño cheese aged for 15 days and $60 \%$ of fresh costeño cheese, while the shelf life was approximately 23 days.
\end{abstract}

Keywords: utilization; shelf life; processed cheese; costeño cheese; aging. 


\section{INTRODUCCIÓN}

El queso Costeño es un producto autóctono que se produce y distribuye en la región Caribe colombiana (Departamentos de Córdoba, Sucre, Bolívar, Atlántico, Magdalena, Cesar y la Guajira), su producción es artesanal a partir de leche no pasteurizada, con coagulación enzimática, prensado, no madurado, ni acidificado, de color blanco, que se somete a un período de salado de dos horas si este se hace en salmuera o directamente si es el método de salado en seco y elaborado bajo las mínimas condiciones higiénicas sanitarias, por lo que su consumo masivo aumenta el riesgo de intoxicación alimentaria (Soto-Varela et al., 2018). Existe una alta demanda por éste producto, ya que posee un sabor y aroma agradable y característico, los que son atribuidos a la actividad metabólica microbiana autóctona que presenta la leche cruda. Su vida útil es corta, debido al uso de leche cruda y ausencia de maduración lo que conlleva a acelerar el crecimiento y proliferación de bacterias que causa defectos en el sabor y en el olor, favoreciendo la pérdida de sus características organolépticas, dificultando su comercialización a gran escala (Queiroz et al., 2017; RuízPérez et al., 2017). Una forma de aprovechar el queso costeño envejecido, que ha perdido sus características típicas es utilizarlo como materia prima en el proceso de elaboración de queso procesado o fundido tipo untable, de forma que se produzca un alimento con características fisicoquímicas, microbiológicas y sensoriales que cumplan con la normatividad disponible, sea aceptado por el consumidor y contribuir con ello ha disminuir el riesgo de enfermedades transmitidas por alimentos. Es una alternativa viable para disminuir las pérdidas de este producto nutritivo y autóctono de la región, considerando que de 6.900 millones de litros de leche producidos en Colombia en el año 2017, aproximadamente el $26 \%$ se dedican a la fabricación de los quesos regionales (Costeño, Caqueteño y Paipa) (Fedegan, 2015; 2018).

El queso procesado o fundido se ha convertido en una buena alternativa para las industrias lácteas, debido a que tiene un período de vida útil prolongado y presenta mejor facilidad de manejo en las empresas. Se obtiene mezclando, agitando y calentando hasta fundir una mezcla de quesos crudos, con sales emulsionantes, agua y limitada cantidad de ingredientes adicionales (Fu et al., 2018). La mezcla de quesos puede ser de quesos naturales de la misma o diferente variedad y en diferentes grados de madurez. Para la producción del queso procesado untable, es necesario que en su formulación exista la combinación de quesos jóvenes, medianamente madurados y muy maduros para obtener una textura adecuada (Fox et al., 1998). Los quesos madurados presentan texturas largas y suaves, ya que las caseínas se hidrolizan durante la maduración, por lo que la extensión del queso será corta y untable (Bejarano-Toro et al., 2016). Bejarano-Toro et al. (2016) realizaron una investigación en donde utilizaron queso antioqueño como materia prima para elaborar un queso procesado, el queso antioqueño es un queso fresco al que le adicionaron Bifidobacterium bifidum almacenándolo durante 25 días para incentivar la hidrolisis de la caseína, y obtener así, un queso procesado con características fisicoquímicas y sensoriales adecuadas.

El objetivo de esta investigación, fue obtener un queso procesado untable a partir de queso costeño fresco y envejecido sin adición de bacterias, determinar sus características fisicoquímicas, microbiológicas, sensoriales y el tiempo de vida útil.

\section{METODOLOGÍA}

Los quesos fundidos fueron elaborados en el taller de Procesos lácteos de la Planta Piloto de la Universidad de Córdoba, ubicado en el corregimiento de Berástegui en Córdoba, Colombia. Se utilizó como sal fundente una mezcla de citrato de sodio, fosfato disódico y fosfato trisódico aportado por TECNAS S.A de Colombia. Para estandarizar la formulación del queso fundido se utilizó, agua potable y mantequilla (Colanta, Colombia).

\section{Elaboración de queso costeño}

La metodología que se utilizó para la obtención del queso costeño, se basó en el proceso descrito en el Manual de elaboración de Queso Costeño Amasado (ICTA, 1986). Las etapas realizadas fueron; Ajuste de temperatura de la leche $\left(30-32^{\circ} \mathrm{C}\right)$, adición de $\mathrm{CaCl}_{2}(15 \mathrm{~g} / 100 \mathrm{~L})$ y cuajo industrial $(2.5 \mathrm{~g} / 100 \mathrm{~L})$, coagulación por 30 minutos, corte de la cuajada, agitación de la cuajada, desuerado, salado ( $2.5 \%$ sal), prensado, empaque y almacenamiento $\left(4-6^{\circ} \mathrm{C}\right)$.

\section{Formulación y obtención del queso fundido}

Para la obtención del queso fundido se utilizó una mezcla de queso costeño fresco y quesos envejecidos durante 15 y 30 días, almacenados a $5+1{ }^{\circ} \mathrm{C}$. Se tomó como referencia para el cálculo de la formulación, la composición de la materia prima (Queso costeño), formulaciones establecidas en la literatura y los rangos especificados por la Norma Técnica Colombiana (NTC) 4225 (1997). La metodología para la elaboración del queso fundido untable, se basó en el proceso descrito por Erazo (2012) con algunas modificaciones. Las etapas realizadas fueron; selección y limpieza superficial de los quesos costeños, troceado, macerado, 
mezclado (adición de sales fundentes, agua y grasa, de acuerdo a los tratamientos), fundido (temperatura de $85^{\circ} \mathrm{C}$ durante 13 minutos en un tanque enchaquetado con agitación (Javar Tecnología alimentaria, Colombia), empacado y almacenamiento $\left(5 \pm 1^{\circ} \mathrm{C}\right)$. Los tratamientos utilizados se presentan en la Tabla 1 . En la Tabla, Q0: queso costeño fresco; Q15: queso costeño envejecido por 15 días; Q30: queso costeño envejecido por 30 días.

Tabla 1: Tratamientos establecidos

\begin{tabular}{|c|c|c|c|}
\hline Tratamientos & Q0 (\%) & Q15 (\%) & Q30 (\%) \\
\hline 1 & 40 & 60 & - \\
\hline 2 & 50 & 50 & - \\
\hline 3 & 40 & - & 60 \\
\hline 4 & 50 & - & 50 \\
\hline
\end{tabular}

De acuerdo a los tratamientos establecidos se obtuvieron las diferentes cantidades de materia prima e ingredientes a utilizar (la cantidad de sal fundente adicionada fue del $2.5 \% \mathrm{p} / \mathrm{p}$ del queso costeño utilizado) de acuerdo a la NTC 4225 (1997), luego de realizar la caracterización fisicoquímica de la materia prima, se procedió a calcular la composición final de la mezcla. La formulación establecida fue de $60 \%$ de humedad y $40 \%$ de materia seca. La composición de las formulaciones según los tratamientos se presenta en Tabla 2.

Tabla 2: Composición de las formulaciones por tratamiento

\begin{tabular}{|l|c|c|c|c|}
\hline \multirow{2}{*}{ Materias Primas } & \multicolumn{4}{|c|}{$\%$ Total $(\mathrm{g} / 100 \mathrm{~g})$} \\
\cline { 2 - 5 } & $\mathrm{T} 1$ & $\mathrm{~T} 2$ & $\mathrm{~T} 3$ & $\mathrm{~T} 4$ \\
\hline Q0 & 20.11 & 25.04 & 20.11 & 25.04 \\
\hline Q15 & 29.83 & 25.04 & - & - \\
\hline Q30 & - & - & 29.83 & 25.04 \\
\hline Mantequilla & 14.92 & 14.96 & 14.92 & 14.96 \\
\hline $\begin{array}{l}\text { Mezcla de sal } \\
\text { fundente }\end{array}$ & 1.25 & 1.25 & 1.25 & 1.25 \\
\hline Agua & 33.88 & 33.70 & 33.88 & 33.70 \\
\hline Mezcla total & 100 & 100 & 100 & 100 \\
\hline
\end{tabular}

\section{Análisis Fisicoquímico de la leche, materia prima y queso procesado}

Tanto a las muestras de leche, quesos costeños (fresco y envejecido), y el queso procesado tipo untable se les realizó el análisis fisicoquímico que se presenta en la Tabla 3.

\section{Análisis microbiológicos del queso procesado}

El recuento de mohos y levaduras, mesófilos aerobios en placas con agar en siembra profunda y coliformes totales y fecales por el número más probable; se determinaron teniendo como referencia a Vanderzant y Splittstoesser (1992).

\section{Selección de las formulaciones de mayor aceptación}

Se evaluó la aceptación general de los quesos fundidos, utilizando una escala hedónica de 7 puntos (7: Me gusta muchísimo, 1: Me disgusta muchísimo) con 50 jueces no entrenados (De Paula et al., 2014), se seleccionaron los dos tratamientos con mayor aceptación.

\section{Determinación del tiempo de vida útil}

A los dos tratamientos seleccionados en el punto anterior, se les determinó la vida útil en tiempo real, utilizando el límite de aceptabilidad microbiana (LAM) a través de la ecuación de Gompertz parametrizada, determinando el día en que la concentración de mesófilos aeróbios alcanzó el umbral de $5 \times 10^{4}$ UFC/g según la NTC 4225 (1997). La temperatura empleada para determinar la vida útil fue la de su conservación comercial $(5 \pm 1 \stackrel{\circ}{ } \mathrm{C})$. El conteo fue realizado 2 veces por semana (cada 3 y 7 días).

\section{Análisis estadístico}

Para obtener los diferentes tratamientos, se usó un diseño experimental completamente al azar. Los datos se procesaron mediante análisis de varianza y Test de Tukey utilizando el software Statgraphics Centurion $X V I I I \circledR$. 
Tabla 3: Análisis para evaluar la leche, queso costeño y procesado

\begin{tabular}{|l|l|l|l|}
\hline Muestras & \multicolumn{1}{|c|}{ Análisis } & \multicolumn{1}{|c|}{ Método } & Referencia (AOAC, 2005) \\
\hline \multirow{3}{*}{$\begin{array}{l}\text { Leche y } \\
\text { Queso }\end{array}$} & $\mathrm{pH}$ & Potenciométrico & Peláez et al.(2003) \\
\cline { 2 - 4 } & Acidez & Titulación & 920.124 \\
\cline { 2 - 4 } & Humedad & Gravimétrico & 948.12 \\
\cline { 2 - 4 } & Materia grasa & Babcock modificado & 989.04 \\
\hline \multirow{4}{*}{$\begin{array}{l}\text { Queso } \\
\text { procesado }\end{array}$} & Proteína & Kjedahl modificado & 920.123 \\
\cline { 2 - 4 } & Cenizas & Calcinación por vía seca & 923.03 \\
\cline { 2 - 4 } & Cloruros & Volumetría & 16.272 \\
\cline { 2 - 4 } & Humedad & Gravimétrico & 948.12 \\
\cline { 2 - 4 } & Materia grasa & Babcock modificado & 989.04 \\
\cline { 2 - 4 } & pH & Potenciométrico & Peláez et al.(2003) \\
\hline
\end{tabular}

\section{RESULTADOS Y DISCUSIÓN}

Se presentan los resultados obtenidos bajo las condiciones de este trabajo. Los resultados se muestra en forma separada para cada tipo de análisis: caracterización fisicoquímica de la leche, caracterización fisicoquímica de la materia prima, caracterización fisicoquímica del queso fundido, caracterización microbiológica del queso fundido, análisis sensorial del queso fundido, y determinación del tiempo de vida útil del queso fundido.

\section{Caracterización fisicoquímica de la leche}

La composición fisicoquímica de la leche utilizada como materia prima para la elaboración del queso costeño se presenta en la Tabla 4. En la Tabla, Leche Q0: es leche cruda para obtener queso costeño fresco; Leche Q15: es leche cruda para el queso costeño envejecido por 15 días; Leche Q30: es leche cruda para obtener queso costeño envejecido por 30 días. De acuerdo con los resultados obtenidos de \% de grasa, \% de sólidos totales, acidez y pH, para Leche Q0, Leche Q15 y Leche Q30, se puede decir que todos los valores están dentro de los límites establecidos por la legislación colombiana (Decreto 616, 2006).

Tabla 4: Características fisicoquímicas de la leche utilizada para la obtención del queso costeño. *Valores medios en triplicado con la misma letra en cada columna no son diferentes estadísticamente por la prueba de Tukey $(p>0,05)$.

\begin{tabular}{|c|c|c|c|c|}
\hline Muestras de Leche & Grasa (\%) & Sólidos Totales (\%) & Acidez (\% ácido láctico) & $\mathrm{pH}$ \\
\hline Leche Q0 & $4.15 \pm 0.07^{\mathrm{a}}$ & $12.26 \pm 0.66^{\mathrm{a}}$ & $0.18 \pm 0.01^{\mathrm{a}}$ & $6.47 \pm 0.01^{\mathrm{a}}$ \\
\hline Leche Q15 & $3.13 \pm 0.04^{\mathrm{b}}$ & $11.30 \pm 0.06^{\mathrm{a}}$ & $0.17 \pm 0.01^{\mathrm{a}}$ & $6.60 \pm 0.01^{\mathrm{b}}$ \\
\hline Leche Q30 & $2.65 \pm 0.07^{\mathrm{c}}$ & $11.36 \pm 0.06^{\mathrm{a}}$ & $0.18 \pm 0.01^{\mathrm{a}}$ & $6.58 \pm 0.03^{\mathrm{b}}$ \\
\hline
\end{tabular}

\section{Caracterización fisicoquímica de la materia prima}

Los resultados del análisis fisicoquímico de los quesos utilizados como materia prima se presentan en la Tabla 5. En el contenido de grasa de los quesos utilizados como materia prima para la elaboración del queso fundido, existen diferencias estadísticamente significativas, siendo Q0 y Q15 iguales, sin embargo si se hace una relación con la grasa de la leche utilizada para elaborar estos quesos (Tabla 4), se debería obtener diferencia estadística, ya que el contenido de grasa en la leche utilizada para la elaboración de los quesos, presenta diferencias estadísticas, la grasa es el componente más variable en la leche y es al mismo tiempo la que más cambios sufre por efecto genético, fisiológico y nutricional.

Tabla 5: Parámetros fisicoquímicos del queso costeño. *Valores medios en triplicado con la misma letra en cada columna son iguales estadísticamente, prueba de Tukey $(p>0.05)$

\begin{tabular}{|c|c|c|c|c|}
\hline Queso costeño & Grasa (\%) & Materia seca (\%) & Acidez (\% ácido láctico) & $\mathrm{pH}$ \\
\hline Q0 & $25.37 \pm 0.12^{\mathrm{a}^{*}}$ & $51.28 \pm 0.08^{\mathrm{a}}$ & $0.62 \pm 0.01^{\mathrm{c}}$ & $6.24 \pm 0.01^{\mathrm{c}}$ \\
\hline Q15 & $25.30 \pm 0.06^{\mathrm{a}}$ & $52.92 \pm 0.64^{\mathrm{a}}$ & $0.81 \pm 0.01^{\mathrm{b}}$ & $5.83 \pm 0.03^{\mathrm{b}}$ \\
\hline Q30 & $21.03 \pm 0.13^{\mathrm{b}}$ & $53.02 \pm 0.07^{\mathrm{b}}$ & $1.25 \pm 0.02^{\mathrm{a}}$ & $5.67 \pm 0.01^{\mathrm{a}}$ \\
\hline
\end{tabular}

Posiblemente el almacenamiento de los quesos influye directamente en la composición final del Q15; de igual manera lo plantearon Golin et al. (2018), quienes en su trabajo fabricaron queso Coalho y evaluaron las propiedades fisicoquímicas durante el almacenamiento obteniendo un aumento del contenido de grasa durante el transcurso del mismo, esto debido a la disminución del contenido de humedad por causa de la liberación del lactosuero y al aumento de la acidez en el almacenamiento. Sin embargo, el Q30 presenta un contenido inferior de grasa $(p<0.05)$ que el Q0 y Q15; debido posiblemente al bajo nivel de grasa en la leche utilizada para su elaboración. 
Para la variable materia seca, el análisis estadístico indicó que hubo diferencia significativa entre los valores promedios de los quesos ( $p<0.05$ ), siendo el Q15 y Q0 iguales. El Q30 es el de mayor contenido en materia seca, debido posiblemente a que un mayor tiempo de almacenamiento provoca mayor pérdida de humedad (Golin et al., 2018). Para la variable pH se presentó diferencias estadísticamente significativas entre todos los quesos $(p<0.05)$. La diferencia en el $\mathrm{pH}$, posiblemente se debe a cambios provocados por la flora natural fermentativa presente en los quesos, que por permanecer por tiempos diferentes en almacenamiento, sufrió cambios tanto en el pH como en la acidez, así como en el color y olor.

Acevedo et al. (2015) reportaron una media de pH de 5.30 en queso costeño amasado, valores que están por debajo de los obtenidos en este estudio. La acidez obtenida varió entre $0.62 \%$ y $1.25 \%$ AL., según el análisis estadístico se observa que hubo diferencia significativa entre los valores promedios obtenidos en todos los quesos $(p<0.05)$. El aumento de la acidez con el almacenamiento genera mayor contracción de la cuajada, y por lo tanto una mayor expulsión de lactosuero de la misma (Simpson et al., 2012), esto fue demostrado con el aumento del porcentaje de materia seca en la medida que fue incrementado el tiempo de envejecimiento (Tabla 5). Acevedo et al. (2015) reportaron un valor promedio de acidez de $0.60 \%$ para queso Costeño, un valor similar fue obtenido para el queso costeño sin envejecimiento (Q0).

\section{Caracterización fisicoquímica del queso fundido}

Los resultados promedios del análisis fisicoquímico del queso fundido untable se muestran en la Tabla 6 . El análisis estadístico de $\mathrm{pH}$ del queso fundido, mostró que hubo diferencias significativas entre todos los tratamientos $(p<0.05)$. Esta diferencia pudo haberse dado porque el nivel de proteína en cada tratamiento fue diferente lo que influye en los valores finales del pH o también a la capacidad buffer de las sales fundentes (Hosseini-parvar et al., 2014). Los valores obtenidos están por encima de los reportados por Salek et al. (2016) en el intervalo de 5.61 a 5.78 en queso fundido tipo suizo con diferentes grados de maduración y por los resultados reportados por Hosseini-parvar et al. (2014) en el intervalo de 5.36 a 5.66 en queso procesado untable de caseína de cuajo. El pH final de queso procesado es un factor importante que controla la estructura final y por lo tanto las propiedades funcionales finales del queso procesado (Hosseini-parvar et al., 2014). Para un queso procesado tipo untable el pH óptimo oscila entre 5.6 a 6.0 (Buňka et al., 2012), este pH brinda características de fundido y emulsificación adecuadas, los valores de $\mathrm{pH}$ de los diferentes tratamientos se encontraron dentro del rango del pH óptimo, indicando buena calidad en el queso procesado.

Tabla 6: Parámetros fisicoquímicos del queso fundido tipo untable a partir del queso costeño. *Valores en triplicado con la misma letra en cada columna son iguales estadísticamente, prueba de Tukey $(p>0.05)$

\begin{tabular}{|c|c|c|c|c|c|c|}
\hline Tratamiento & $\mathrm{pH}$ & Humedad (\%) & Proteína (\%) & Grasa (\%) & Cloruros (\%) & Cenizas (\%) \\
\hline T1 & $5.93 \pm 0.01^{\mathrm{b}^{\mathrm{a}}}$ & $42.00 \pm 0.63^{\mathrm{c}}$ & $15.16 \pm 0.78^{\mathrm{b}}$ & $39.00 \pm 1.38^{\mathrm{a}}$ & $1.66 \pm 0.03^{\mathrm{b}}$ & $3.06 \pm 0.10^{\mathrm{a}}$ \\
\hline T2 & $5.95 \pm 0.01^{\mathrm{a}}$ & $44.00 \pm 0.63^{\mathrm{c}}$ & $16.98 \pm 0.16^{\mathrm{a}}$ & $38.87 \pm 0.29^{\mathrm{a}}$ & $1.37 \pm 0.04^{\mathrm{c}}$ & $1.03 \pm 0.04^{\mathrm{c}}$ \\
\hline T3 & $5.78 \pm 0.01^{\mathrm{d}}$ & $54.33 \pm 2.25^{\mathrm{a}}$ & $13.86 \pm 0.82^{\mathrm{c}}$ & $35.05 \pm 0.71^{\mathrm{b}}$ & $1.75 \pm 0.04^{\mathrm{a}}$ & $2.74 \pm 0.06^{\mathrm{b}}$ \\
\hline T4 & $5.89 \pm 0.01^{\mathrm{c}}$ & $46.66 \pm 1.03^{\mathrm{b}}$ & $14.96 \pm 0.68^{\mathrm{b}}$ & $37.35 \pm 0.21^{\mathrm{a}}$ & $1.38 \pm 0.03^{\mathrm{c}}$ & $2.72 \pm 0.04^{\mathrm{b}}$ \\
\hline
\end{tabular}

Para la variable humedad, en la Tabla 6, se puede apreciar que los tratamientos T1 (40\% Q15 - 60\% Q0) y T2 (50\% Q15 - $50 \%$ Q0) son iguales estadísticamente, mientras que los tratamientos T3 (40\% Q30-60\% Q0) y T4 (50\% Q30 - $50 \%$ Q0) presentan diferencias estadísticas. Estas diferencias se pudieron presentar debido a la variabilidad del contenido de materia grasa, humedad, porcentaje de mezcla, tiempo de envejecimiento del queso Costeño utilizado como materia prima e igualmente al pH final, presencia de iones por las sales fundentes utilizadas y el proceso de manufactura del queso fundido (Da Silva et al., 2016). Giri et al. (2014) encontraron valores de humedad de $56 \%$ y $58 \%$, en queso untable tipo Cheddar con fitosteroles, por su parte, Hosseini-parvar et al. (2014) reportaron valores de humedad entre $52-56 \%$, en queso untable de caseína de cuajo. Por otro lado, los valores promedios de proteína de los tratamientos presentaron diferencias significativas, siendo los tratamientos T1 y T4 iguales estadísticamente, mientras que los tratamientos T2 y T3 diferentes entre todos $(p<0.05)$. Estas diferencias entre los tratamientos se pudieron presentar por los diferentes valores de grasa, humedad y proteína de la materia prima y/o al tipo y porcentaje de queso costeño en cada tratamiento. Lee et al. (2015) obtuvieron un comportamiento similar en diferentes quesos fundidos con diferentes contenidos de proteína y materia grasa.

Los valores de la grasa en los tratamientos variaron entre 35.05 y $39.00 \% \quad(p<0.05)$, estos valores están por encima de los reportados por Rubel et al. (2019) en un intervalo de $30.78 \%$ a $33.50 \%$ para queso fundido untable tipo Ricotta. Los cloruros variaron entre $1.37 \%$ y $1.75 \%(p<0.05)$, Giri et al. (2014), investigaron sobre el efecto de los fitosteroles en las características texturales y de fusión de queso fundido para untar, reportaron valores para el porcentaje de cloruros de $1.9 \%$ y $2 \%$. Los tratamientos T1 (40\% Q15 - 60\% Q0) y T3 (40\% Q30 - 60\% Q0) presentan $1.66 \pm 0.03 \%$ y $1.75 \pm 0.04 \%$ de cloruros, respectivamente, los que se encuentran en el rango mencionado por este autor. Los niveles bajos de cloruros encontrados en la investigación se deben a que no hubo adición de cloruro de sodio en el proceso de fundido, ya que la materia 
prima utilizada presenta el $2.5 \%$ de cloruro de sodio, además hubo un proceso de adición de agua, lo cual influyó en el la disminución del contenido de cloruros en el producto final. Por último, los datos encontrados arrojaron que el contenido de cenizas varió entre $1.03 \pm 0.04 \%$ y $3.06 \pm 0.0 \%$. El análisis estadístico muestra que existen diferencias significativas $(p<0.05)$ entre tratamientos siendo los tratamientos T3 y T4 iguales entre sí, y T1 y T2 totalmente diferentes entre los tratamientos. Valores superiores fueron encontrados por Giri et al. (2014).

\section{Caracterización microbiológica del queso fundido}

La Tabla 7, muestra los valores promedios de los recuentos microbiológicos del queso fundido en los diferentes tratamientos. Los tratamientos analizados fueron estadísticamente iguales $(p>0.05)$ y no presentaron coliformes totales y fecales, lo cual indicó que no hubo contaminación de los quesos por manipulación durante el proceso de manufactura del mismo, ya que se mantuvieron las condiciones de sanidad en todas las etapas. De acuerdo con la NTC 4225 (1997) para quesos fundidos, el valor máximo permitido para identificar nivel de buena calidad es menos de $3 \mathrm{NMP} / \mathrm{g}$, por tanto, se puede decir que los tratamientos estudiados cumplieron con este requisito. Por otro lado, el análisis estadístico del conteo de mohos y levaduras de los cuatro tratamientos estudiados mostró que hubo diferencia significativa $(p<0.05)$, los mayores recuentos lo presentaron los tratamientos T3 (40\% Q30 - $60 \%$ Q0) y T4 (50\% Q30 - $50 \%$ Q0) que fueron iguales estadísticamente, pero diferentes a los tratamientos T1 (40\% Q15-60\% Q0) y T2 (50\% Q15 - $50 \%$ Q0) $(p<0.05)$, posiblemente la causa de la diferencia entre los tratamientos fueron algunas características intrínsecas del producto como por ejemplo la humedad (Tabla 6). Los valores reportados estuvieron por debajo de los encontrados por Costa et al. (2017) con un valor promedio de 2 Log UFC/g en queso tipo untable. En general todos los tratamientos estuvieron por debajo del índice máximo permisible para indicar nivel de buena calidad (2.30 Log UFC/g) de acuerdo con la NTC 4225 (1997).

Tabla 7: Resultados del análisis microbiológico del queso fundido tipo untable a partir del queso costeño. * Valores medios en triplicado con la misma letra en cada columna son iguales estadísticamente por la prueba de Tukey $(p>0.05)$

\begin{tabular}{|c|c|c|c|}
\hline Tratamiento & $\begin{array}{c}\text { Coliformes totales y E. coli } \\
\text { (NMP/g) }\end{array}$ & $\begin{array}{c}\text { Mohos y levaduras (Log } \\
\text { UFC/g) }\end{array}$ & $\begin{array}{c}\text { Mesófilos aerobios (Log } \\
\text { UFC/g) }\end{array}$ \\
\hline T1 & $<3^{a^{*}}$ & $1.11 \pm 0.16^{c}$ & $2.09 \pm 0.04^{\mathrm{c}}$ \\
\hline T2 & $<3^{\mathrm{a}}$ & $1.34 \pm 0.16^{\mathrm{b}}$ & $2.19 \pm 0.04^{\mathrm{b}}$ \\
\hline T3 & $<3^{\mathrm{a}}$ & $1.54 \pm 0.16^{\mathrm{a}}$ & $2.48 \pm 0.03^{\mathrm{a}}$ \\
\hline T4 & $<3^{\mathrm{a}}$ & $1.54 \pm 0.16^{\mathrm{a}}$ & $2.48 \pm 0.03^{\mathrm{a}}$ \\
\hline
\end{tabular}

Finalmente, para el conteo de mesófilos aerobios, se observó la misma tendencia que en el conteo de mohos y levaduras. Los tratamientos evaluados mostraron valores promedios en el intervalo de 2.09 a 2.48 Log UFC/g $(p<0.05)$. Estos valores se encuentran dentro del intervalo reportado por Kim et al. (2018) con conteos promedios en torno de 1.15 a $5.14 \mathrm{Log}$ UFC/g en diferentes tipos de quesos procesados.

\section{Análisis sensorial del queso fundido}

El valor promedio del nivel de aceptación de los diferentes tratamientos por parte de los consumidores es presentado en la Tabla 8. A través de la evaluación sensorial se logró establecer que los tratamientos T1 (40 $\%$ Q15 - 60\% Q0) y T4 (50\% Q30 - $50 \%$ Q0) no presentaron diferencia significativa $(p>0.05)$ y fueron los más aceptados por los panelistas con valores medios superiores a 5 (en la escala de 1 a 7 ). En este sentido, este estudio presenta una contribución tecnológica relevante de la utilización de queso costeño como materia prima para la fabricación de queso procesado tipo untable.

\section{Determinación del tiempo de vida útil del queso fundido}

La Figura 1, reporta la evolución de mesófilos aerobios para los tratamientos que fueron los más aceptados. El mejor ajuste de la ecuación propuesta a los datos experimentales es presentado en la Figura 1; la línea paralela al eje de las abscisas muestra el valor límite permisible para indicar nivel aceptable de calidad según la NTC 4225 (1997) para mesófilos aerobios. El límite de aceptabilidad microbiana (LAM) representa el tiempo en días en el cual el queso fundido no es microbiológicamente aceptable (Costa et al., 2017). Los valores medios de LAM para el tratamiento T1 fue de $23.93 \pm 1.10$ días y para el tratamiento T4 de $21.13 \pm 0.55$ días, siendo diferentes estadísticamente $(p<0.05)$. Esta diferencia pudo acontecer debido a las propiedades intrínsecas de los tratamientos, como la humedad y el contenido de sal, que de alguna u otra forma generan gran influencia en el crecimiento de algunos microorganismos (Borowik y Wyszkowska, 2016). Los valores de LAM obtenidos en este estudio fueron superiores a los encontrados por Costa et al. (2017), con valores promedios de $5.59 \pm 0.32$ días para queso untable. Restrepo \& Montoya (2010) establecen que este período depende de muchas variables en donde se incluyen tanto el producto como las condiciones ambientales y el empaque. 
Tabla 8: Aceptación del queso fundido tipo untable por parte de los consumidores *Valores medios con la misma letra no son diferentes estadísticamente por el prueba de Tukey $(p>0,05)$

\begin{tabular}{|c|c|}
\hline Tratamiento & Valor de aceptación \\
\hline T1 & $5.36 \pm 0.28^{\mathrm{a}^{*}}$ \\
\hline T2 & $4.81 \pm 0.39^{\mathrm{b}}$ \\
\hline T3 & $4.80 \pm 0.37^{\mathrm{b}}$ \\
\hline T4 & $5.29 \pm 0.39^{\mathrm{a}}$ \\
\hline
\end{tabular}

En la Fig. 1 se puede apreciar que el conteo de mesófilos aerobios presentó un incremento gradual hasta sobrepasar el límite permisible para indicar nivel aceptable de calidad (5000 UFC/g). De acuerdo con la Tabla 5, el tratamiento T1 presentó un menor contenido de humedad y una mayor concentración de sal, lo que pudo dar como resultado una menor tasa de crecimiento microbiana comparado con el tratamiento T4, y por consiguiente un mayor tiempo de vida útil $(23.93+1.10$ días). En general, el estudio de vida útil de los dos tratamientos arrojó datos importantes para la investigación, ya que siendo productos sin la adición de conservantes artificiales, alcanzaron valores de vida útil superiores a 3 semanas.

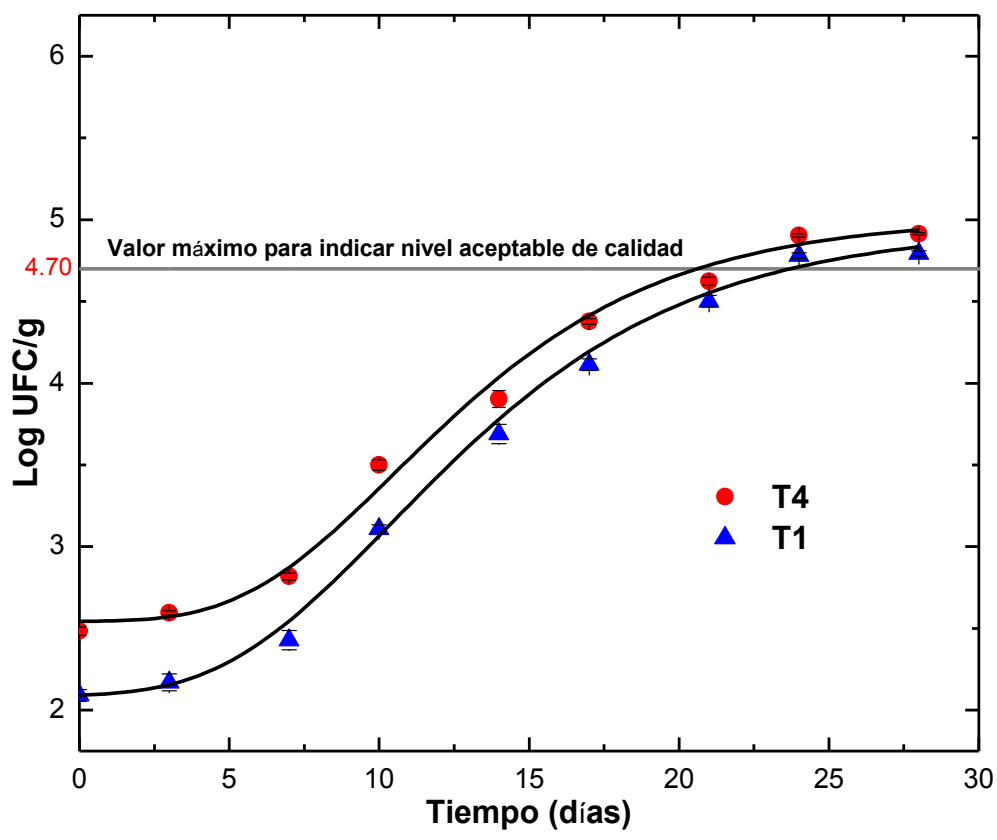

Fig. 1: Proliferación de mesófilos aerobios en el tiempo para los tratamientos 1 y 4.

\section{CONCLUSIONES}

De acuerdo a los resultados obtenidos, se puede concluir que fue posible obtener queso fundido tipo untable a partir de queso costeño fresco $(40 \%)$ y envejecido $(60 \%)$ durante 15 días de almacenamiento a $5{ }^{\circ} \mathrm{C}$; el cual tuvo una buena aceptación sensorial y un tiempo de vida útil de 23.93 días. Las características fisicoquímicas y microbiológicas del queso fundido para untar cumplen con los parámetros establecidos por la Norma Técnica Colombiana 4225, permitiendo elaborar un producto de buena calidad.

\section{REFERENCIAS}

Acevedo, D., Jaimes J.D y Espitia C.R., Efecto de la Adición de Lactosuero al Queso Costeño Amasado, Información Tecnológica, 26(2), 11-16 (2015).

A.O.A.C, Official Methods of Analysis, 18th E. AOAC, Arlington V.A, USA (2005).

Bejarano-Toro, E. E., Sepúlveda-Valencia J. U. y Restrepo-Molina D. A., Characterization of a Processed Cheese Spread Produced from Fresh Cheese (Quesito Antioqueño), Rev. Fac. Nacional de Agronomía, 69(2), 8015-8022 (2016).

Borowik, A y Wyszkowska J., Soil moisture as a Factor affecting the Microbiological and Biochemical Activity of Soil, Plant Soil Environ, 62(6), 250-255 (2016).

Buňka, F., Doudová L. y otros cinco autores, The Effect of Different Ternary Mixtures of Sodium Phosphates on Hardness of Processed Cheese Spreads. Int. Journal of Food Science \& Technology, 47(10), 2063-2071 (2012). 
Costa, C., Lucera A. y otros tres autores, Application of Preservation Strategies to Improve the Shelf life of Spreadable Cheese, Food Packaging and Shelf Life, 11, 16-20 (2017).

Da Silva, D y otros tres autores, Effect of Commercial Konjac Glucomannan and Konjac Flours on Textural, Rheological and Microstructural Properties of Low Fat Processed Cheese, Food Hydrocolloids, 60, 308-316 (2016).

Decreto 616. Requisitos que debe cumplir la leche para el consumo humano que se obtenga, procese, envase, transporte, comercializa, expenda, importe o exporte en el país, 1-32, Ministerio de la Protección Social, Bogotá D.C, Colombia (2006).

De Paula, C. D., García C.A. y Durango A.M., Valor de la Marca en la Aceptación Sensorial de Consumidores en cuatro tipos de Pan Tajado Blanco, Información Tecnológica, 25(1), 127-132 (2014).

Erazo, L, Elaboración de Queso Fundido Untable tipo Cheddar en Industria Lechera Carchi S.A, Tesis de grado, Facultad de Ciencias e Ingeniería de Alimentos, Universidad Técnica de Ambato, Ambato, Ecuador (2012).

Fedegan, Cifras de Referencia del Sector Ganadero Colombiano del segundo semestre (en la web: http://www.fedegan.org.co/estadisticas/general, acceso 6 de mayo de 2019), FEDEGAN, Colombia (2018)

Fedegan, Concentración del Mercado de la Leche (en la web: http://static.fedegan.org.co/Revistas_Carta_Fedegan/150/ 14ANALISIS_ECONOMICO.pdf, acceso 6 de mayo de 2019), FEDEGAN, Colombia (2015)

Fox, P. F., Uniacke-Lowe T., McSweeney P. L. H y O'Mahony J. A., Dairy Chemistry and Biochemistry, 2a ED., 421-427, Springer International Publishing, Suiza (1998)

Fu, W., Watanabe Y. y otros seis autores, Effects of Pre-cooked Cheeses of Different Emulsifying Conditions on Mechanical Properties and Microstructure of Processed Cheese, Food Chemistry, 24(5), 47-52 (2018).

Giri, A., Kanawjia S.K. y Rajoria A., Effect of Phytosterols on Textural and Melting Characteristics of Cheese Spread, Food Chemistry, 157, 240-245 (2014).

Golin, R., Costa B. y otros cinco autores, Manufacture of Reduced-sodium Coalho Cheese by Partial replacement of NaCl with $\mathrm{KCl}$, International Dairy Journal, doi: 10.1016/j.idairyj.2018.07.012 (2018).

Hosseini-parvar, S. H., Matia-merino L. y Golding M., Effect of Basil Seed Gum (BSG) on Textural, Rheological and Microstructural Properties of Model Processed Cheese, Food Hydrocolloids, 1-11 (2014).

ICTA, Instituto de Ciencia y Tecnología de Alimentos, Manual de Elaboración de Queso Costeño Amasado. 1a ED, 1939, Universidad Nacional de Colombia, Colombia (1986).

Kim, N. H., Lee N.Y. y otros cinco autores, Microbiological criteria and Ecology of commercially Available Processed Cheeses according to the Product Specification and Physicochemical Characteristics. https://doi.org/10.1016/j.foodres.2018.01.014, Food Research International, 106 (January), 468-474 (2018)

Lee, S. K., Klostermeyer H. y Anema S.G., Effect of Fat and Protein-in-water Concentrations on the Properties of Model Processed Cheese, International Dairy Journal, 50, 15-23 (2015).

NTC 4225. Productos Lácteos. Quesos Fundidos. Requisitos Específicos, ICONTEC, 6-7, Bogotá D.C, Colombia (1997)

Queiroz, M. M y otros tres autores, Hygienic-sanitary quality of Minas Fresh Cheese sold in the city of Botucatu, São Paulo, Arquivos Do Instituto Biológico, 84(0) (2017).

Peláez, P y otros tres autores, Caracterización Físico-química de Quesos Frescos elaborados con Leche de cabra en la Isla de Tenerife, Cienc. Tecnol. Aliment, 4(2), 103-108 (2003)

Restrepo, A y Montoya C., Implementación y Diseño de procedimiento para Determinación de Vida útil de Quesos Frescos, Chorizos Frescos y Aguas en Bolsa, Tesis de grado, Facultad de Tecnologías, Univ. Tecnológica de Pereira, Pereira, Colombia (2010)

Rubel, I. A., Iraporda C. y otros tres autores, Spreadable Ricotta Cheese with Hydrocolloids: Effect on Physicochemical and Rheological Properties, https://doi.org/https://doi.org/10.1016/j.idairyj.2019.03.002, Int. Dairy Journal, (2019)

Ruíz-Pérez, R. A., Menco-Morales N.Y. y Chams-Chams L. M., Valoración Microbiológica de Queso Costeño Artesanal y Evaluación Higiénico-locativa de expendios en Córdoba, Colombia Microbiological evaluation of artisan coastal cheese and hygienic- locative evaluation of small shops in Córdoba, Colombia, Rev. Salud Pública, 19(3), 311-317 (2017).

Salek, R. N., Černíková M. y otros tres autores, The Effect of Different Composition of Ternary Mixtures of Emulsifying Salts on the Consistency of Processed Cheese Spreads manufactured from Swiss-type Cheese with different Degrees of Maturity, J. Dairy Sci 99, 1-14 (2016).

Simpson, B. K., Nollet L.M., Toldrá F., Benjakul S., Paliyath S. y Hui Y.H., Food Biochemistry and Food Processing, 2a ED., 442-462, Wiley-Blackwell (2012)

Soto-Varela, Z. E., Gutiérrez C.G. y otros cuatro autores, Detección molecular de Salmonella spp., Listeria spp. y Brucella spp. en Queso Artesanal Fresco comercializado en Barranquilla: un estudio piloto. Biomédica, 38, 30-36 (2018).

Vanderzant, C y Splittstoesser D.F., Compendium of Methods for the Microbiological Examination of Foods. 1-1219, American public health association, USA (1992). 